\title{
Fuerza pragmática y carácter institucional del lenguaje: entre la acción comunicativa y el poder simbólico
}

\section{Pragmatic force and language's institutional character: between communicative action and symbolic power}

JAVIER ALEGRE

Doctor en Filosofía - Docente-investigador en el área de Filosofía Contemporánea Institución: Universidad Nacional del Nordeste (UNNE) - Argentina

E-mail: pillancho@yahoo.com.ar

\begin{abstract}
Resumen
El presente artículo toma como trasfondo las elaboraciones teóricas sobre el lenguaje realizadas por Wittgenstein y Austin y aborda en plan comparativo la manera en que las propuestas de Habermas y Bourdieu reelaboran los hallazgos teóricos de aquella perspectiva pragmática original. En particular, nos interesa analizar el modo en que el carácter institucional atribuido al lenguaje es retomado en la teoría de la acción comunicativa de Habermas y la pragmática sociológica de Bourdieu, con el propósito de mostrar y confrontar dos modos posibles, distintos e irreductibles entre sí, de apropiarse de la fuerza heurística que yace en el giro pragmático inaugurado por Wittgenstein y Austin a mediados del siglo XX.
\end{abstract}

\begin{abstract}
This article takes Wittgenstein and Austin's reflections about language as background and it presents comparatively the way Habermas and Bourdieu rebuild that original pragmatic perspective's theoretic finds. We are specifically interested in analyzing the way in which the institutional character of language is recovered by Habermas's communicative action theory and Bourdieu's sociological pragmatic, in order to show and to confront two different and irreducible manners of appropriate the heuristic power of pragmatic turn started by Wittgenstein and Austin in the middle of XX century.
\end{abstract}


- Palabras claves: Pragmatismo lingüístico - Habermas - Bourdieu - Racionalidad Violencia simbólica

- Key words: Linguistic Pragmatism - Habermas - Bourdieu - Rationality - Symbolic Violence

La participación del lenguaje en la constitución de las prácticas sociales y su relación con las instituciones han sido enfocadas desde diversas perspectivas, el trasfondo teórico del presente artículo proviene del pragmatismo lingüístico contemporáneo de mediados del siglo XX, más precisamente utilizamos como plataforma los aportes teóricos provistos por la segunda etapa filosófica de Wittgenstein y la teoría de los actos de habla de Austin (cfr: Wittgenstein 2004, 1991; Austin 1970, 1962). Ahora bien, nuestro propósito no es hacer una exégesis y discutir hacia adentro las propuestas teóricas de ambos pensadores fundacionales, sino analizar y confrontar las reelaboraciones y redireccionamientos que sus legados encuentran en los planteos de dos autores ligados en sentido amplio al giro pragmático, Jürgen Habermas y Pierre Bourdieu, quienes presentan aspectos divergentes de importancia en lo que respecta al modo de entender el carácter pragmático e institucional del lenguaje.

En las reapropiaciones de Habermas y Bourdieu se expresa una preocupación por incorporar permanentemente los procesos simbólicos que guían las acciones intersubjetivas, así como por acompañar la fuerza heurística del pensamiento pragmático con los aportes de otras tradiciones teóricas. En Habermas como en Bourdieu hay una superación del modelo intencionalista en lo que refiere a la explicación del sentido y las acciones simbólicas, en este aspecto ambos se mantienen fieles a las convicciones pragmáticas de Wittgenstein y Austin y reconocen la ascendencia de las convenciones sociales y semánticas en la conformación de las intenciones de los sujetos, los significados y las interacciones. Ahora, es claro que las reflexiones de Habermas y Bourdieu responden a intereses diferentes y toman orientaciones disímiles, de lo cual se desprenden consecuencias muy divergentes. El presente artículo está dedicado precisamente a explorar y confrontar estos contrapuntos y explicitar sus incumbencias.

En particular, aquí nos proponemos abordar las elaboraciones de Habermas y Bourdieu sobre el lenguaje con el objetivo de, por un lado, evaluar sus reapropiaciones de 
la multiplicidad pragmática y la gran capacidad realizativa del lenguaje señaladas por Wittgenstein y Austin y, por el otro lado, ponderar el modo en que recuperan el carácter institucional del lenguaje y si en este intento logran recoger con éxito la potencia heurística inicial del giro pragmático. En pos de cumplir estos propósitos, hemos estructurado el artículo en tres partes: a) una primera, en que analizamos el pragmatismo universal y la teoría de la acción comunicativa de Habermas y su visión consensualista del lenguaje; b) una segunda, en que nos abocamos a la pragmática sociológica del constructivismo estructuralista de Bourdieu y su visión no consensualista del lenguaje; y c) una tercera y última, en que hacemos un análisis crítico-confrontativo de los puntos centrales y elaboramos nuestras reflexiones finales.

\section{a) Habermas: la acción comunicativa como apuesta pragmática para afrontar los procesos de institucionalización modernos}

En las elaboraciones teóricas de Habermas el bagaje conceptual proveniente de las reflexiones iniciadas por el giro pragmático está puesto en relación con un amplísimo abanico de corrientes que ligan con un contexto de discusión filosófica y sociológica mucho más extendido -conceptual y temporalmente- que el del pragmatismo lingüístico. La propuesta habermasiana se caracteriza por ser una crítica de la racionalización mediante el despliegue de una teoría de la racionalidad en términos reconstructivos, o dicho de manera grandilocuente: una crítica de la razón para defender y salvar a la razón -de otras críticas más devastadoras-. Para Habermas los problemas de racionalización y cosificación, que son de su interés central, pertenecen a la línea alemana del pensamiento que sigue el derrotero Kant-Hegel-Marx-Weber-Lukács y desemboca en la escuela de Frankfurt; por ello, lo que Habermas intenta es renovar y reconducir ciertos aspectos de esta amplia línea teórica a partir de los aportes del pragmatismo lingüístico, al que recurre para salir del punto muerto al que habría conducido la crítica hiperbólica frankfurtiana y así recuperar los aspectos positivos del proyecto de racionalización moderna (cfr: Habermas 2003a, 2003b, 1994a, 2003c, 2008).

En este marco, la significación de los actos de habla depende de su orientación a lograr el acuerdo entre los participantes de la comunicación y el lenguaje es considerado como el ámbito donde se puede lograr el consenso entre los integrantes de una comunidad 
que en última instancia tiene alcance universal. Habermas defiende un concepto de racionalidad inherente a la intersubjetividad, surgida en los procesos de interacción comunicativa de los sujetos socializados y poseedora de un carácter situado, ya no omnipotente (cfr: Habermas 1990a, pp. 38-63). El sustrato desde el cual se origina la acción humana racional no es el sujeto o la conciencia -en cuanto garantes metafísicos del conocimiento o la verdad - sino una intersubjetividad que posee carácter lingüístico: las experiencias y el mundo son inseparables del horizonte que traza el lenguaje en nuestra compresión. La actividad lingüística tiene un carácter meta-institucional y se convierte en la más propia del género humano, ${ }^{1}$ y esta actividad, a su vez, tiene una función que le es inherente y predomina sobre cualquiera de las otras funciones que puede llevar a cabo: el entendimiento.

La pragmática universal habermasiana tiene por objetivo la reconstrucción sistemática de las estructuras generales de los actos de habla, pero, además, persigue el establecimiento de las condiciones de posibilidad para el logro de todo acuerdo posible en el lenguaje ordinario, de allí que sea un estudio reconstructivo y normativo en términos universales; ${ }^{2}$ "la pragmática universal tiene como tarea identificar y reconstruir las condiciones universales del entendimiento posible (...). Parto, pues (...) de que otras formas de acción social, por ejemplo, la lucha, la competencia y en general el comportamiento estratégico, pueden considerarse derivados de la acción orientada al entendimiento" (Habermas 1994a, p. 299). Así, la teoría de la acción comunicativa (TAC) se configura como la propuesta mediante la cual Habermas responde a un doble objetivo reconstructivo: por un lado, la reconstrucción de las competencias comunicativas y pretensiones de validez presentes en los actos de habla; y, por el otro, la reconstrucción de la lógica evolutiva que guía los procesos de modernización social occidentales (cfr: Habermas 1981, pp. 9-44 y 131-180). Abordada desde el punto de vista habermasiano -y

\footnotetext{
${ }^{1}$ Si bien se infiere el carácter meta-institucional del lenguaje de sus diferentes textos, Habermas lo deja explícito en un temprano artículo de 1967 en un tramo en que discute la concepción del lenguaje en Gadamer: "no es descabellado entender el lenguaje como una especie de metainstitución de la que dependen todas las instituciones sociales. Pues la acción social sólo se constituye en la comunicación en el lenguaje ordinario" (Habermas 1988, p. 257).

${ }^{2}$ Reconstructivo en cuanto busca reconstruir las presuposiciones necesarias para el funcionamiento del lenguaje a través de un análisis pragmático-formal dirigido a los usos y competencias comunicativas, haciendo centro en el lenguaje como habla (como proceso, no como estructura) y en la acción orientada al entendimiento. Y normativo porque establece cuáles condiciones y pretensiones deben estar presentes para que se cumpla el entendimiento (considerado uso original y fin propio del lenguaje), por lo que esas condiciones y pretensiones son constituyentes de la acción comunicativa y, a su vez, sirven como criterio de evaluación acerca del logro o no del entendimiento.
} 
expresándolo de modo esquemático-, la TAC puede entenderse nada más, y nada menos, que como el resultado de la conjunción de estos dos amplios objetivos teóricometodológicos en relación con el interés por una sociedad emancipada en la que se integren la acción y la auto-determinación solidarias de sus integrantes.

La vinculación entre lenguaje y procesos de institucionalización presenta una doble imbricación según Habermas: el lenguaje contribuye a través de sus usos a los procesos de institucionalización de las diferentes esferas de acción y, a su vez, la institucionalización de la racionalidad moderna especifica las funciones del lenguaje y libera sus potencialidades comunicativas. Así, el interés de Habermas está puesto en la relación específica entre el lenguaje y las instituciones legadas por la modernidad, es decir, en la interrelación entre la función del lenguaje como reaseguro de la racionalidad ya destrascendentalizada y los procesos de institucionalización que son producto de la modernización occidental. Habermas deja en claro que para asegurar niveles crecientes de racionalización es necesario que se formen y consoliden nuevas instituciones que se estructuren a partir de las esferas comunicativas del mundo de la vida. La posibilidad de erigir las instituciones que contrarresten la colonización producida por los sistemas de acción está en total dependencia de la capacidad del lenguaje de coordinar las acciones a través del entendimiento y el acuerdo, es decir, de la institucionalización de ámbitos en que dicha capacidad pueda plasmarse, lo que significa la institucionalización de la racionalidad comunicativa. Por ello, son las instituciones del mundo de la vida las que actúan como soporte necesario de los procesos de institucionalización de la racionalidad (cfr: Habermas $2003 b) .^{3}$

La acción comunicativa desempeña una función trascendental para Habermas, no en el sentido que sus presuposiciones y condiciones sean inviolables, sino que de no cumplirlas se incurre inevitablemente en formas patológicas o distorsionadas de

\footnotetext{
${ }^{3}$ Según Habermas si bien la dinámica evolutiva (modo efectivo en que se desarrollaron las formaciones y hechos históricos concretos con su dinámica propia) de la modernización ha conducido a la colonización del mundo de la vida y sus consecuencias patológicas, la lógica evolutiva (lógica de desarrollo de neto corte formal que puede abstraerse del desarrollo histórico y que se expresa en estructuras cognitivo-racionales) de la diferenciación estructural moderna no implica necesariamente que deba desembocarse en ellas; la dinámica podría darse de otra manera, para no ser patológica debería recurrirse a las potencialidades racionales y autónomas de la acción comunicativa que han sido liberadas también por el proceso de modernización. La conocida defensa del legado y las posibilidades abiertas por la modernidad presente en diferentes textos de Habermas dio lugar a numerosas publicaciones, dentro de éstas resultan particularmente ricas - por la profundidad y cercanía investigadora de sus participantes-: Honneth, McCarthy, Offe y Wellmer 1992a; Honneth, McCarthy, Offe y Wellmer 1992b; Passerin y Benhabib 1997.
} 
comunicación. La acción comunicativa se desempeña aquí como medio de institucionalización de la racionalidad y como mecanismo de integración y coordinación social por excelencia, no existen otros usos del lenguaje o elementos que puedan cumplir estas funciones de modo similar ya que carecen de las condiciones y capacidades estructurales dirigidas al entendimiento, cooperación y solidaridad; "mientras sólo se utilice el lenguaje como medio para la transmisión de informaciones y de «redundancias», la coordinación de la acción discurre a través del influenciamiento mutuo de actores que operan los unos sobre los otros con el fin de conseguir cada uno sus propios fines. Pero en cuanto las fuerzas ilocucionarias de los actos de habla asumen un papel coordinador de la acción, es el lenguaje mismo el que aparece como fuente primaria de integración social. Sólo en este caso ha de hablarse de «acción comunicativa»" (Habermas 1998, p. 79).

Así, el lenguaje, antes que medio de transmisión de informaciones, es fuente de integración social y tiene por paradigma las actividades comunicativas orientadas al entendimiento, las cuales se distinguen de los usos estratégicos del lenguaje básicamente por presentar pretensiones universales de validez susceptibles de fundamentación y crítica (verdad, rectitud y veracidad) y poseer interés emancipatorio (cfr: Habermas 2003a). Si bien Habermas otorga carácter de interacción auténtica tanto a la acción estratégica como a la acción comunicativa, establece una división y confrontación tajantes entre ambos modelos al punto de reservar para la acción comunicativa todas las propiedades ligadas a la ampliación de la racionalidad y la emancipación y entender la acción estratégica sólo como una forma defectiva de aquella, comprensible únicamente a partir de la carencia de determinados elementos constituyentes de la primera y ligada a instancias de coacción para la imposición de intereses particulares: "obtenemos así el modelo de interacción estratégica cuando, partiendo del caso de acción comunicativa, vamos eliminando por pasos todas las presuposiciones del empleo del lenguaje orientado al entendimiento, (...) en la medida en que las interacciones no quedan coordinadas a través del entendimiento, la única alternativa es la violencia que los unos ejercen contra los otros (...). No es otra cosa lo que quiere decir la distinción tipológica entre acción comunicativa y acción estratégica" (Habermas 1994a, pp. 456-9). Por ello, Habermas mantiene estrictamente enfrentados los planos de la racionalidad comunicativa y la racionalidad con arreglo a fines, de la acción comunicativa y la acción estratégica, del mundo de la vida y el mundo sistémico, aunque 
reconoce que ambos tipos de acción se presentan generalmente en forma conjunta y que sólo es válido -y conveniente- separarlos en el análisis (cfr: Habermas 1990b, p. 309).

Así, Habermas entiende la acción estratégica como una acción social que se presenta como un caso límite de la acción comunicativa, ya que es a la que se recurriría cuando en el lenguaje no se da el reconocimiento de normas intersubjetivamente válidas, por lo que ya no podría servir como medio de entendimiento y pasaría a ser utilizado como un medio de influir o disuadir a los demás hablantes en base a una actitud egocéntrica y objetivante. Ambos tipos de acción se presentan como irreductibles y enfrentados en el plano teórico y como alternativas incompatibles para los participantes en el plano práctico. Es evidente que una confrontación tan notoria entre ambas esferas de acción, antes que atender a la forma efectiva en que se entablan las acciones lingüísticas, responde más bien a las exigencias teóricas que se le presentan a la TAC, que necesita reservar el uso fundamental y prototípico del lenguaje sólo para las acciones orientadas al entendimiento y hacer depender de ellas toda posible ampliación de los niveles de estructuración racional y emancipación de la sociedad. Al colocar en la base de su concepción del lenguaje la distinción entre las esferas de acciones estratégicas y acciones comunicativas, Habermas cuenta con una herramienta eficaz para hacer notar que el progreso técnico no equivale al aumento de comportamientos racionales ni de relaciones sociales libres de dominación, pero esta distinción deviene en división abrupta y oposición lineal que dificulta la comprensión del entramado que ambas esferas de acción conforman en las prácticas cotidianas e introduce desde el inicio una idealización y una separación abismal que imposibilitan que cualquier tipo de interconexión no sea vista como perniciosa y avasallante en los niveles posteriores de análisis en que estas acciones participan (interacción y trabajo, mundo de la vida y sistemas de acción, sociedad civil y esfera productiva, etc.).

La concepción del lenguaje de Habermas tiende a hipostatizar la función del entendimiento, ya que ésta es colocada como criterio de base para comprender y evaluar todo acto de habla: cualquier acción lingüística será más o menos válida en cuanto incorpore en mayor o menor medida los elementos propios del habla comunicativa orientada al entendimiento, estipulando la acción comunicativa como único modelo válido de acción lingüística y concibiendo como desviación patológica a toda institucionalización surgida de la modernidad que no esté exclusivamente sustentada en y estructurada por el 
modelo universal del habla comunicativa. Como Habermas considera que las acciones comunicativas son el único criterio válido para el análisis y comprensión de las demás, los otros tipos de acción se ven reducidos a modelos defectivos de interacción, ligados a generalizaciones indebidas en base a acciones de validez y racionalidad parcializadas; ante lo cual se vuelve problemático el hecho que el lenguaje en su totalidad -no sólo el uso comunicativo- contribuye a la institucionalización de los distintos ámbitos de racionalidad (cognitivo-instrumental, estético-expresivo y jurídico-moral) y a que es posible institucionalizar los diversos usos del lenguaje, no únicamente el comunicativo. Al tomar para sus análisis una función del lenguaje como la única función paradigmática y válida de modo excluyente (los demás tipos aparecen como formas defectivas y desviadas de la función comunicativa), Habermas comete con el lenguaje, aunque en dirección conceptual inversa, una sinécdoque similar a la que había atribuido a los primeros frankfurteanos: tomar un tipo de razón, la instrumental, como la forma de racionalidad moderna.

Si bien Habermas entiende, en sintonía con la perspectiva pragmática, la intersubjetividad y la capacidad realizativa como componentes básicos del análisis del lenguaje, sus elecciones metodológicas y la hipostatización de la función comunicativa del lenguaje conducen a lo que podemos denominar como depotenciación crítico-pragmática. Depotenciación originada en considerar la pragmática lingüística sólo en términos formales-reconstructivos y retomar las dos principales fuentes de la TAC (la teoría crítica y el pragmatismo lingüístico, tradiciones en las que se inscribe y de las que, al mismo tiempo, se distancia) de tal modo que sus indagaciones quedan desprovistas de herramientas metodológicas y conceptuales acordes para abordar la manera en que los factores comunicativos interactúan con las condiciones contextuales, lo que dificulta indudablemente el establecimiento de una conexión más estrecha entre su vastísimo registro teórico y el contexto de situaciones e intereses no discursivos. La TAC toma como único modelo a los actos de habla en que se cumplen las pretensiones universales de validez y, por lo tanto, se desinteresa mayoritariamente del entrelazamiento de estas formas linguiísticas idealizadas con las demás formas de acción e interacción y prescinde de analizar en profundidad las condiciones fácticas que hacen posible la realización de los procesos discursivos, por lo que las conexiones entre su bagaje conceptual y las situaciones y usos reales se ven notoriamente debilitadas y la pragmática lingüística queda encorsetada en líneas formales reconstructivas que la orientan con exclusividad hacia un uso 
discursivo-deliberativo del lenguaje sólo dable en esferas limitadas que, además, no dependen para su instauración sólo de factores discursivos sino también de factores extradiscursivos y del modo en que éstos se integran con las prácticas discursivas.

Como colofón de este primer apartado, realizamos algunas puntualizaciones acerca del modo específico en que Habermas retoma las teorías de Wittgenstein y Austin.

La influencia wittgensteniana en las reflexiones de Habermas queda de manifiesto especialmente en vincular la racionalidad al ámbito de la lingüísticidad y la intersubjetividad, remitir el sentido a la capacidad basal de seguir reglas comunitariamente -no a la esfera de la intencionalidad ni de la conciencia privada- y oponerse a la existencia de una razón trascendente y de procesos de argumentación o crítica con pretensiones de fundamentación última. Además, también para Habermas el lenguaje ordinario es el ámbito de intersubjetividad que hace posible la interacción, el entendimiento y la comprensión mutua y debido a su reflexividad constituye su propio meta-lenguaje y es el marco de referencia para la interpretación del mundo histórico. Asimismo, Habermas encuentra en Wittgenstein las elaboraciones pioneras en dos puntos de suma importancia para el armado de su propio sistema teórico: a) la inclusión de la dimensión pragmática del empleo de las oraciones en la teoría del significado como uso, y b) la sustitución del paradigma de la conciencia por el del lenguaje, con la consecuente reducción de la importancia de la intencionalidad en favor de las propiedades del lenguaje.

Ahora bien, Habermas considera que el fin inmanente del lenguaje es el entendimiento y la producción de acuerdos discursivos y para ello se basa en las reflexiones de Wittgenstein: “con Wittgenstein estoy convencido que «lenguaje»y «entendimiento» son conceptos cooriginarios, conceptos que se explican mutuamente" (Habermas 1994a, p. 417), o bien: "Wittgenstein se ha percatado de que el concepto de acuerdo reside en el concepto de lenguaje. (...) Todo acuerdo se acredita, tal y como nosotros decimos, en un consenso racional; de lo contrario no es ningún acuerdo «real»" (Habermas 1987, p. 29). Pero en esta reapropiación es posible identificar un error fundamental: para el segundo Wittgenstein el concepto de acuerdo no reside en el lenguaje, sino, por el contrario, el funcionamiento del lenguaje únicamente puede ser entendido en base a los acuerdos ya dados en las formas de vida compartidas, son éstas las que sirven de fundamento a los acuerdos reflejados en el lenguaje o, como sumo, que el lenguaje ayuda a conformar. Para Wittgenstein, en todo caso, los que son cooriginarios son acuerdo y 
formas de vida, justamente la posibilidad de entendimiento y consensos dentro del lenguaje está basado en la coincidencia de formas de vida. ${ }^{4}$ Habermas identifica todo consenso con el cumplimiento de las pretensiones universales expresadas paradigmáticamente en la situación ideal de habla, pero con esto produce una reducción lingüística de los consensos a todas vistas indebida si partimos de los principios wittgensteinianos acerca de que el consenso puede expresarse en el lenguaje, pero no necesariamente parte del lenguaje y de sus pretensiones de validez. Esta interpretación forzada de Wittgenstein no es casual dentro de las reflexiones habermasianas, sino que está emparentada con su afán de establecer la prioridad de la función del entendimiento por sobre cualquier otra y su erección como único modelo válido de acción lingüística.

En lo que respecta a la teoría de los actos de habla, Habermas la toma como fundamento para sus elaboraciones sobre el lenguaje y al acto de habla como unidad básica de la comunicación. Ahora, si bien se basa en Austin y Searle, sostiene que éstos todavía se encuentran influidos por la perspectiva de la semántica filosófica y, por lo tanto, se aparta de su comprensión en diversos puntos. Para elaborar su TAC, Habermas corrige parcialmente la distinción entre actos ilocucionarios y perlocucionarios introducida por Austin, ya que entiende que la persecución del fin perlocucionario de ejercer influencias a través de un acto de habla únicamente puede darse si se cumple el fin ilocucionario de que sea comprendido lo dicho; aquí los actos perlocucionarios/estratégicos están subsumidos en los actos ilocucionarios/comunicativos, por lo que los efectos perlocucionarios se corresponden con una clase de interacciones estratégicas pues se encuentran dentro del uso teleológico del lenguaje. En vista de ello es que para el entendimiento dado en la acción comunicativa únicamente deban considerarse aquellos actos en que los hablantes persiguen nada más que fines ilocucionarios, es decir que los múltiples efectos ilocucionarios del lenguaje, propuestos por Austin, dejan aquí su sitio al modelo único del entendimiento; “cuento, pues, como acción comunicativa aquellas interacciones mediadas lingüísticamente en que todos los participantes persiguen con sus actos de habla fines ilocucionarios y sólo fines ilocucionarios. Las interacciones, en cambio, en que a lo menos uno de los participantes pretende con sus actos de habla provocar efectos perlocucionarios en su interlocutor, las considero como acción estratégicamente mediada lingüísticamente" (Habermas 2003a, p. 378).

\footnotetext{
${ }^{4}$ Cfr. especialmente: Wittgenstein 2004, §241, §23; Wittgenstein 1991, §559.
} 


\section{b) Bourdieu: la fuerza pragmática del lenguaje y las condiciones sociales de su rendimiento simbólico}

Bourdieu no se preocupa por brindar una teoría general del lenguaje, sino que se aboca a presentar una teoría de las prácticas e intercambios lingüísticos, a partir de la cual sí se pueden establecer los principios que constituyen su concepción del lenguaje. La clave de su aproximación teórica está dada por el entrelazamiento entre prácticas linguiísticas y demás prácticas sociales y la relación que aquellas presentan con la distribución desigual de capitales y las luchas -tanto de fuerza como simbólicas- entabladas en torno de ello. Así, se plasma en el ámbito lingüístico su visión no consensualista y agonística de todas las prácticas, además de su oposición a la filosofía de la conciencia y al reinado de las categorías cognitivas ligadas a la noción de sujeto (donde retoma la crítica austiniana a la scholastic view enunciada en Sense and Sensibilia). En la teoría bourdieusiana, el campo lingüístico presenta los mismos rasgos que los demás campos en base a la homología estructural que comparten y, de modo congruente con el giro pragmático, el agente se hace junto con las prácticas lingüísticas, entre otras prácticas, lo cual desplaza los fundamentos mentales y representacionales en favor de los componentes relacionales y disposicionales y los condicionamientos sociales. La vinculación constante entre los elementos del mundo lingüístico-simbólico y los del mundo social hace que toda relación de fuerza sea también una relación simbólica y, viceversa, toda fuerza debe ser reconocida para que actúe eficazmente y todo reconocimiento simbólico se basa en la dispar distribución de fuerzas dada en el espacio social (cfr: Bourdieu 1980, 1982, 1997, 1994). ${ }^{5}$

La concepción agonística, historicista e inmanente que Bourdieu atribuye a la esfera social, y por lo tanto también a las prácticas lingüísticas, hace que el lenguaje se presente como factor de comunicación y conocimiento tanto como de poder y dominación. A través del lenguaje se persiguen beneficios simbólicos dado el valor social y la eficacia simbólica

\footnotetext{
${ }^{5}$ Esta relación indisoluble entre lo lingüístico y lo social vuelve patente el carácter histórico, dinámico e inmanente de las acciones y producciones lingüísticas y conduce a que Bourdieu critique los planteos de corte ahistoricistas, que asocia especialmente con Saussure y Habermas; "no existen, sin duda, por más que piense en ello Habermas, universales transhistóricos de la comunicación; pero existen ciertamente formas socialmente constituidas de comunicación que favorecen la producción de lo universal" (Bourdieu 2005, p. 126). En relación con esto, para Calhoun la diferencia principal entre Habermas y Bourdieu, más allá de ciertas similitudes que encuentra, está en el rechazo de Bourdieu a las aproximaciones de tipo teorético encarnadas en la TAC y en el desinterés de Habermas por el dominio práctico y las estrategias de los agentes en los procesos sociales (cfr: Calhoun 1993).
} 
que posee, por lo que el análisis del lenguaje debe tener en cuenta la estrecha conexión que éste mantiene con las condiciones y relaciones sociales en que se da. Todo lenguaje porta jerarquización y dominación, por eso aquí ocupan un lugar primordial las funciones de legitimación y reproducción de las relaciones de fuerzas presentes en el espacio social relaciones que tienen expresión en el lenguaje-, de aquí que se centre especialmente en la economía de las prácticas lingüísticas y en el modo que éstas contribuyen a la dominación simbólica, política y económica.

El lenguaje posee una lógica práctica que no se guía exclusiva ni primordialmente por principios economicistas ni de cálculo racional, sino que refiere a prácticas que se realizan sin la necesidad de ser efectivamente conscientes o dirigirse a objetivos preestablecidos; lo que predomina es la comprensión incorporada en los habitus de los agentes y objetivada en las instituciones. El habitus lingüístico forma parte del habitus como sistema general de disposiciones constitutivas, por lo que resulta de la introyección generada por la posición, condiciones y relaciones del agente en el espacio social, es el producto de la transformación de las necesidades y presiones grupales en elecciones y virtudes individuales y está incardinado férreamente en el cuerpo, "el lenguaje es una técnica del cuerpo y la competencia propiamente lingüística, y muy especialmente la fonológica, es una dimensión de la hexis corporal donde se expresan toda la relación del mundo social y toda la relación socialmente instruida del mundo" (Bourdieu 1982, pp. 8990). El cuerpo y el habitus están doblemente ligados al lenguaje y las instituciones, tanto porque las disposiciones incorporadas del habitus son las que mantienen vivos y revitalizan los sentidos depositados en el lenguaje y las instituciones, como porque las estrategias de adquisición del poder simbólico emanante de la legitimidad de las prácticas linguísticas e institucionales toma la forma del sentido práctico con que los agentes se desenvuelven en y utilizan el lenguaje y las instituciones.

El lenguaje participa en la integración cognoscitiva y social a un orden desigual y desempeña un papel importante en la sumisión dóxica necesaria para la eficaz tarea de la dominación simbólica, dominación caracterizada por presentarse en forma deformada, eufemizada e irreconocible y que al no ser reconocida como tal puede trabajar libremente y obtener su mayor rendimiento. Esta dominación se impone gracias a las condiciones estructurales que favorecen a los grupos dominantes, pero para llevarse a cabo necesita de la adhesión y aceptación de los grupos dominados, es en esta dimensión simbólica donde el 
orden establecido adquiere sentido, "la dominación simbólica (...) se basa en el desconocimiento y por lo tanto en el reconocimiento de los principios en nombre de los cuales se ejerce" (Bourdieu 1994, p. 187). Por ello, para Bourdieu, todas las relaciones cognitivas y comunicativas son a la vez relaciones de violencia y de poder simbólicos en tanto que en ellas se plasman relaciones inequitativas entre los integrantes de la sociedad, relaciones desiguales que quedan disimuladas, ocultas y justificadas tras el carácter compartido, multidireccional y legitimado de los intercambios simbólicos.

Con esto, Bourdieu se opone a las teorías de corte comunicacional, las que ve representadas principalmente por Austin, Searle y Habermas, y las busca complementar a través de la incorporación del entramado social del que surge la fuerza ilocucionaria de las palabras. Así, la performatividad de una emisión está en dependencia aquí del poder delegado que posee quien la enuncia, si el lenguaje funciona es porque existe un reconocimiento -implícito, olvidado, irreconocible- de la autoridad y superioridad del emisor, por lo que es infructuoso postular, aunque sea idealmente, situaciones abstractas de igualdad y consenso en el lenguaje. Al portar el lenguaje jerarquización y sumisión, cuanto más establecida e institucionalizada esté la jerarquía con que cuenta el emisor, mayor será la eficacia simbólica de su enunciación; Bourdieu lo expresa casi en tono de ley física: "la eficacia del discurso performativo que pretende hacer advenir lo que enuncia en el acto mismo de enunciarlo es proporcional a la autoridad de quien lo enuncia” (Bourdieu 1982, pp. 140-1).

La eficacia simbólica surge de la relación entre las propiedades del discurso, del portavoz, de la institución que lo autoriza a pronunciarlo y de la configuración de fuerzas del espacio social, con esto Bourdieu subraya el carácter institucional de los actos realizativos y la importancia de las condiciones sociales en el funcionamiento del lenguaje. ${ }^{6}$ El poder simbólico está incardinado en el reconocimiento institucional de las palabras y, principalmente, del emisor indicado a partir de relaciones y distribuciones desiguales, y no en el propio lenguaje ni en las fuerzas ilocucionarias por sí mismas: "el poder simbólico como poder de constituir lo dado por la enunciación, de hacer ver y de hacer creer, de confirmar o de transformar la visión del mundo y, por ello, la acción sobre

\footnotetext{
${ }^{6}$ Igual vale recalcar que Bourdieu no reduce toda la capacidad simbólica del lenguaje a su actuación institucional; si bien la performatividad del lenguaje encuentra en las condiciones institucionalizadas su mejor campo de acción, también puede darse su funcionamiento sobre bases menos sólidas, aunque esto siempre restringirá su eficacia y necesitará de una posterior institucionalización para consolidar su efecto performativo (cfr: Bourdieu 1979, p. 560).
} 
el mundo (...) no reside en los "sistemas simbólicos" bajo la forma de una "illocucionary force", sino que se define en y por una relación determinada entre los que ejercen el poder y los que lo sufren, es decir, en la estructura misma del campo donde se produce y se reproduce la creencia" (Bourdieu 2005, pp. 71-2). ${ }^{7}$

La Realpolitik de la razón de la que es partidario Bourdieu toma la forma, dentro del campo lingüístico, de Realpolitik del lenguaje e intercambios simbólicos, posicionamiento mediante el cual Bourdieu resalta la necesidad de ir contra la jerarquización y dominación presentes en el lenguaje y la presión ejercida por las condiciones asimétricas de adquisición y producción simbólica entre los diferentes agentes. Para esta Realpolitik lingüística, antes que centrarse en la fuerza ilocucionaria de las palabras o plantear situaciones ideales de habla, es necesario actuar políticamente sobre las estructuras sociales objetivas y subjetivas que se presentan como obstáculos específicos a la comunicación racional. La introducción de principios racionales y mayores niveles de equidad en las prácticas lingüísticas no pasa por invocar las potencialidades racionales que puedan yacer en el lenguaje o en las capacidades de sus fuerzas ilocucionarias, sino principalmente por las luchas simbólicas que buscan defender las realizaciones históricas de la razón y el acceso a ellas en mayores condiciones de igualdad; cuestiones en las que, dentro del campo lingüístico, los análisis que integran las estructuras y dinámicas de las relaciones de poder simbólico se encuentran en ventaja respecto de aquellos que las excluyen o minimizan en sus investigaciones. Por ello, Bourdieu aboga por la concreción de una pragmática sociológica dentro de los estudios del lenguaje que conduzca a reincorporar las propiedades formales de los discursos, propiedades que son preocupación excluyente de los estudios analíticos, pero sin recaer en la deformación en que estos abordajes incurrirían por su desatención de los factores sociales que actúan en el lenguaje (cfr: Bourdieu 2001, pp. 328-30). En esta propuesta de pragmática sociológica se evidencian con claridad tanto las preocupaciones de Bourdieu por la inclusión y tratamiento de las condiciones sociales que intervienen en el rendimiento simbólico del lenguaje, en detrimento de los enfoques internalistas y comunicacionales, como el espíritu científico que lo anima en sus indagaciones sociológicas y lingüísticas, en el sentido de dar prioridad y abogar por la

\footnotetext{
${ }^{7}$ Ya en Esquisse d'une théorie de la pratique, en 1972, Bourdieu había asentado con claridad la importancia de los factores extra-discursivos para los análisis lingüísticos (cfr: Bourdieu 2000, p. 246).
} 
ampliación de la autonomía y los efectos del conocimiento científico respecto de su contexto socio-político de producción.

Así, en el análisis de las prácticas lingüísticas de Bourdieu queda la impronta de sus elecciones epistemológicas, realizadas con el propósito de plasmar un sistema teórico que integre la base y fuerza pragmáticas de las expresiones lingüísticas junto con los procesos de violencia y dominación simbólicas que actúan y colaboran en la configuración del espacio social. La clave de la crítica de Bourdieu a los planteos formalistas, analíticos y comunicacionales reside en que estos pasan por alto la dimensión fundamental del lenguaje, en tanto relación de poder simbólico, y lo conciben esencialmente como relación de conocimiento o de comunicación, sin incluir en sus análisis las estructuras y dinámicas propias de las relaciones de poder y la forma transfigurada en que ellas se presentan en las prácticas lingüísticas. Por ello, Bourdieu critica la teoría habermasiana ya que se desinteresa de las condiciones económicas y sociales que posibilitan la instauración de la deliberación pública y la acusa de caer en una ilusión epistemocéntrica al desconocer las condiciones fácticas de acceso e intervención en la esfera pública y de incurrir en una doble reducción indebidamente justificada: primero reduce las relaciones de fuerzas políticas a relaciones de comunicación y, luego, éstas a relaciones exclusivamente de diálogo racional, lo cual da por resultado el desconocimiento de las condiciones sociales y políticas del rendimiento simbólico (cfr: Bourdieu 1997, cap. II). ${ }^{8}$ En resumidas cuentas, las principales críticas de Bourdieu a la TAC se dirigen hacia que es desacertado hacer residir en el lenguaje estructuras universales independientes de las demás prácticas sociales y atribuirles una racionalidad transhistórica; aparte de que la comunicación no es la función paradigmática del lenguaje, sino que éste antes que nada actúa como caja de resonancia de las fuerzas que atraviesan el espacio social.

Para cerrar este segundo apartado, al igual que en el anterior, queremos referirnos en particular a la reapropiación por parte de Bourdieu de sendos autores señeros del giro pragmático. En cuanto a Wittgenstein, es evidente su influencia en la forma anti-mentalista

\footnotetext{
${ }^{8}$ Que el quid de las teorías encontradas de Bourdieu y Habermas sobre el lenguaje reside en la inclusión por parte del primero de la dimensión del poder simbólico en los intercambios lingüísticos es algo de lo que también toma debida cuenta Habermas, quien en una nota al pie señala que los trabajos de Bourdieu representan un tipo de estudio sobre las acciones orientadas al éxito y son un ejemplo de análisis del ejercicio del poder a través del lenguaje, de lo cual se desprende un "concepto de sociedad articulado en términos de teoría del poder" (Habermas 1994a, p. 487); por lo que reduce los análisis de Bourdieu al campo de las acciones estratégicas y, por lo tanto, los encuentra ciegos al mundo de las acciones comunicativas.
} 
y anti-representacionista en que Bourdieu piensa las prácticas sociales y simbólicas y el seguimiento de reglas, clave para la elaboración de su concepto de estrategias. Ahora, Bourdieu no recurre al bagaje conceptual propuesto por Wittgenstein (no hay una utilización específica de conceptos tales como juegos de lenguaje, formas de vida, parecidos de familia), así como tampoco varios ejes de la concepción bourdieusiana del lenguaje se encuentran en los escritos wittgensteinianos (carácter agonístico, relación entre lenguaje, relaciones de poder y fuerzas macro-sociales, etc.). Por lo cual sus reflexiones sobre el lenguaje más que tratarse de una reapropiación de la filosofía wittgensteniana consisten en una continuación sui generis de ella, que no invoca en forma constante su letra sino que se ampara en el espíritu que la imbuye. Esto, al fin de cuentas, lo que hace es exigir una lectura que la entienda más como complementación de la teoría wittgensteiniana antes que como interpretación de ella; tarea de ampliación en la que los puntos de conexión entre ambos autores en algunos casos son fuertes y en otros, mucho más lábiles. ${ }^{9}$

En lo concerniente a la teoría de los actos de habla de Austin, es mucho más clara la bisagra que establece Bourdieu entre aquello que considera apropiado y retoma y aquello que ve como erróneo y desecha de ella. Sus reflexiones sobre el lenguaje se encaraman sobre la capacidad realizativa del lenguaje y la vinculación que ésta posee con la esfera pragmática y con el modo de emplear las emisiones al que recurren habitualmente los hablantes, todos aspectos estudiados originalmente por Austin. Sin embargo, Bourdieu critica en forma reiterada el reduccionismo en que incurriría Austin al centrarse únicamente en los componentes lingüísticos que aseguran el cumplimiento y la eficacia de los actos de habla y descuidar las condiciones sociales (extra-locucionarias) que actúan sobre la fuerza ilocucionaria de las emisiones linguiísticas, "la fuerza ilocucionaria de las expresiones (illocucionary force) no puede encontrarse nunca en las palabras mismas, ni en los «performativos», en los cuales aparece indicada o, mejor, representada (...). Tal es el principio de ese error cuya más cabal expresión nos la proporciona Austin (o Habermas después de él) cuando cree descubrir en el propio discurso, es decir en la sustancia propiamente lingüística -si se nos permite la expresión- de la palabra, su principio de eficacia” (Bourdieu 1982, pp. 103-5). Bourdieu considera que la dirección inicial que guía el proyecto austiniano es correcta y debe ser retomada, pero que hay que apartarse y

\footnotetext{
${ }^{9}$ Charles Taylor, en su conocido artículo "To follow a rule", hace hincapié en los puntos de continuación y realiza una lectura wittgensteiniana de Bourdieu en la que termina asociando indisolublemente ambas teorías (cfr: Taylor 1995).
} 
rectificar el embelesamiento que sufre ante el poder descubierto en las palabras; Austin quedaría preso de una visión ingenua acerca de la capacidad realizativa del lenguaje, que lo lleva a entender los actos de habla como entidades con capacidades cuasi-autónomas y atender sólo a las cuestiones del contexto que atañen en forma directa al funcionamiento de las emisiones lingüísticas particulares.

Ahora bien, esta crítica de Bourdieu no está plenamente justificada ya que Austin en su teoría también contempla e incluye las condiciones sociales e institucionales que deben estar presentes para la concreción exitosa de los actos de habla; no todo el poder de las palabras reside en el propio discurso en la visión austiniana. De todos modos, la preocupación de Austin se dirige casi con exclusividad a las condiciones del contexto que están presentes en forma efectiva en los intercambios lingüísticos o bien a las condiciones institucionales que están en relación directa con ellos; por lo que es posible reconducir la crítica hacia un plano más apropiado señalando que el déficit de la teoría de Austin residiría en no prolongar la atención más allá de las condiciones y convenciones ligadas a determinados factores institucionalizados y grupos sociales particulares, lo que lo lleva a desatender los efectos que causan las fuerzas y procesos sociales más generales sobre el complejo que representa cualquier acto de habla. Las condiciones sociales estructurales necesarias para el eficaz rendimiento del lenguaje tienen una presencia mucho mayor en la teoría de Bourdieu que en la de Austin; al énfasis micro-social, institucional y con ánimos de formalización del primero, se le opone la perspectiva macro-social, agonística y esquiva a los formalismos del segundo. Más allá de estas divergencias, es evidente que a Bourdieu le interesa retener la fuerza pragmática de la teoría austiniana y avanza en la línea abierta por la teoría de los actos de habla, de aquí que haga explícita su admiración y deuda tanto con Austin como con Wittgenstein (cfr: Bourdieu 1987, "Fieldwork in philosophy").

\section{c) Reflexiones finales: en búsqueda de la fuerza pragmática ¿perdida?}

Tanto el pragmatismo universal y la TAC propuestos por Habermas como la pragmática sociológica dentro del constructivismo estructuralista de Bourdieu implican ampliaciones de la perspectiva pragmática inicial hacia nuevos ámbitos y líneas teóricas, claro que dirigidas en diferentes direcciones, por lo que son divergentes sus interpretaciones de los lineamientos del giro pragmático originado en Wittgenstein y 
Austin. Realizamos en este último tramo una breve apreciación crítica de las propuestas de cada autor y luego avanzamos comparativamente en los aspectos centrales abordados en ambas teorías.

En el análisis de las relaciones entre lenguaje e instituciones de Habermas, la introducción en forma decisiva de la intersubjetividad como base y ámbito de la capacidad realizativa del lenguaje y la incorporación de elementos y procesos macro-sociales (sistemas de acción, mundo de la vida, modernización social, etc.) sitúan su abordaje teórico en un nivel mayor de complejidad y amplitud que el que se refleja en el pragmatismo anglosajón, lo que sin dudas provee nuevos relieves y desafíos a los análisis pragmático-institucionales del lenguaje. Ahora, dado que la TAC está dirigida sólo al análisis de los actos de habla propiamente comunicativos, tiende a no atender en forma suficiente los modos en que el lenguaje cumple efectivamente sus muy variadas funciones y a encarar las diferentes dificultades e impedimentos que provienen de estructuras sociales, económicas y políticas muy desiguales desde la óptica exclusiva del incumplimiento de requisitos normativos en las interacciones mediadas lingüísticamente. La perspectiva habermasiana hace retroceder los aspectos fácticos, situacionales e históricos en favor de condiciones ideales y elementos universales ligados a la reconstrucción racional y la teoría formal de la comunicación y considera que la acción comunicativa es el modo original y arquetípico de interacción, llevando hasta tal punto la oposición entre ésta y los demás tipos de acción -reservándoles sustratos, intereses, racionalidades y funcionamientos diferentes- que terminan por aparecer desfiguradas sus posibles interrelaciones, cualquier conexión entre sus esferas se presenta únicamente como avasallante u obstaculizante de los procesos comunicativos. Así, el modo en que realiza su recorte teórico para centrarse sólo en un modo de interacción lingüística, mediante la abstracción e idealización de las condiciones y pretensiones comunicativas, mina la posible potencialidad propositiva de su enfoque respecto de los múltiples usos del lenguaje.

Si bien Habermas reconoce que los distintos tipos de acción están imbricados en la realidad y se encarga en diferentes partes de introducir precisiones respecto del modo en que las prácticas comunicativas toman forma en los procesos sociales tras la modernización, su decisión inicial de encarrilar las indagaciones dentro de un molde reconstructivo formal les quita a éstas buena parte de la fuerza pragmática que podrían obtener por otras vías. Como teoría planteada en términos procedimentales reconstructivos, 
la TAC carece de las herramientas teórico-metodológicas apropiadas para abordar las cuestiones ligadas al establecimiento de las condiciones fácticas que posibilitan que los procesos discursivos se lleven a cabo y para dar cuenta de las limitaciones que poseen los elementos discursivos para explicar su propio surgimiento y aplicación. Habermas mismo reconoce, respecto de este punto crítico, que "ni la disponibilidad ni la capacidad de considerar las cuestiones morales desde la perspectiva a la vez hipotética e imparcial de un participante en discursos prácticos caen del cielo; son resultado de intereses que sólo se forman bajo determinadas condiciones sociales, así como de procesos de formación y de experiencia que sólo son accesibles a los grupos sociales en determinadas situaciones. Lo mismo cabe decir de las nuevas interpretaciones que hacen aparecer nuestras necesidades e intereses a una luz distinta y con ello abren nuevas oportunidades de consenso. Sea cual fuere la forma como surgen estas innovaciones, no alcanzan fuerza de convicción y difusión a través de discursos, sino a través de movimientos sociales" (Habermas 1994a, p. 440). ${ }^{10}$ El carácter netamente crítico-reconstructivo de su teoría es una opción metodológica que realiza en busca de no cargar con las hipotecas que atribuye a la filosofía de la historia y asegurarse el carácter procedimental de la racionalidad que emerge de su TAC, pero imposibilita una conexión más directa entre el amplio bagaje conceptual de su teoría y el entramado de situaciones e intereses en el cual dicho bagaje actúa o está destinado a desempeñarse.

Bourdieu, por su parte, también brinda un análisis en que el lenguaje se abre a los procesos institucionales como elementos necesarios y vitales para concretar sus propiedades realizativas, pero lo vincula directamente con las desigualdades contextuales, el carácter agonístico y la dominación simbólica. Resalta la ineludible relación entre lenguaje e instituciones y el modo en que cada uno refuerza su performatividad en colaboración con el otro: por un lado, el lenguaje adquiere mayor eficacia cuanto más institucionalizadas estén la posición y condiciones de los hablantes y, por el otro, las instituciones logran disimular la violencia simbólica que yace en ellas en buena medida debido a las propiedades del lenguaje. Las relaciones estructurales de poder y fuerzas asimétricas no son entendidas como opuestas a las relaciones lingüísticas, sino que se desempeñan como condición de posibilidad para que el lenguaje actúe en forma normal y

\footnotetext{
${ }^{10}$ En este punto específico, Habermas se está refiriendo a las críticas recibidas por parte de Seyla Benhabib y Steven Lukes en un volumen colectivo dedicado a su teoría (cfr: Thompson y Held 1982).
} 
cumpla eficazmente sus funciones simbólicas; por ello, Bourdieu incorpora en forma permanente la preocupación por la participación del lenguaje en los fenómenos de legitimación y dominación simbólicas. En este punto reside la especificidad de la reapropiación pragmática bourdieusiana, ya que ésta se abre a las relaciones con un contexto social que no queda reducido a los elementos de la intersubjetividad directa ni es visto como un factor distorsionante en base a propiedades asignadas en forma idealizada al lenguaje, sino que intenta combinar los fundamentos teóricos provistos por la perspectiva pragmática con las condiciones y factores micro y macro-sociales que intervienen en la conformación de las prácticas sociales lingüísticas, lo cual también significa reencausar las bases pragmáticas hacia categorías y esferas de mayor amplitud que las usualmente abordadas dentro de la tradición analítico-pragmática.

En correlación con sus intereses teóricos, Habermas encara los estudios sobre el lenguaje con la pretensión de establecer las estructuras y normas racionales, universales, formales y procedimentales que fundarían los procesos sociales, que encuentra expresadas de modo paradigmático en las acciones comunicativas. Bourdieu, por su parte, llega a los estudios sobre el lenguaje a partir de una concepción historicista, sustantiva e inmanente de las acciones sociales, que lo conduce principalmente a la búsqueda de las condiciones y factores extra-locucionarios que hacen posible el funcionamiento y eficacia del lenguaje. Por ello, la aproximación de Habermas exige un distanciamiento de los actos linguí́sticos concretos y una escisión entre la forma de éstos y su contenido y los emisores; mientras que la de Bourdieu hace hincapié en el análisis de los procesos efectivos y vuelve improcedente cualquier intento de establecer divisiones radicales entre las diferentes esferas y tipos de acción y de separar la forma de los actos de habla de las emisiones y hablantes particulares, pues dichos actos conforman un entramado que actúa en conjunto con los mecanismos extra-locucionarios.

Es clave también la dispar apreciación que mantienen ambos acerca de las funciones y alcances de la racionalidad expresada en el lenguaje. Si bien ambos autores comparten la oposición a los enfoques relativistas y anti-racionales (la visión ilustrada, encarnada sobre todo en la teoría habermasiana, no es ajena a la visión bourdieusiana) y defienden y apuestan por la racionalidad propia de las prácticas lingüísticas, lo hacen de manera distinta e irreductible. Habermas, a partir de diferenciar entre la dinámica evolutiva y la lógica evolutiva en los procesos sociales y desligar su procedimiento reconstructivo de la 
dinámica histórica evolutiva, universaliza la razón comunicativa y obtiene estructuras trascendentales para el lenguaje en general, por lo que las condiciones socio-históricas que posibilitan la liberación de las fuerzas racionales ínsitas en los procesos comunicativos quedan en un plano muy secundario frente a la lógica que sigue ese desarrollo y las condiciones idealizadas que actuarían de presupuesto de todo acto de habla. Esta reconstrucción procedimental y universalización de la razón comunicativa es absolutamente ajena a la visión de Bourdieu, quien señala la particularidad y desigualdad propias de los contextos en los que surgen y predominan los intereses y procesos racionales comunicativos. Para Bourdieu en el lenguaje no se encarnan, ni está en dependencia de, estructuras o lógicas transhistóricas independizadas de las condiciones contextuales y sociales, sino que el lenguaje obtiene sus propiedades y funciones de la configuración histórica del espacio social que hace posible tanto la ampliación universal de la racionalidad como la estructuración desigual de los campos y la legitimación de la violencia y la dominación, por lo que aboga por un abordaje histórico de las prácticas sociales, racionales y linguiísticas vinculado con las desigualdades estructurales de sus contextos de desarrollo y utilización y, por lo tanto, opuesto a idealizaciones o divisiones abstractas abruptas. ${ }^{11}$ En este sentido, consideramos que las críticas de Bourdieu son congruentes en buena medida con lo que denominamos como depotenciación críticopragmática en las reflexiones de Habermas -aunque hemos recurrido a líneas de argumentación no análogas-.

En cuanto al carácter institucional del lenguaje, tanto Habermas como Bourdieu proponen análisis en que el lenguaje es una institución íntimamente vinculada con las demás prácticas y procesos institucionalizados, pero también aquí toman caminos bien diferenciados. Según Habermas el lenguaje es una meta-institución comunicativa basada en su capacidad de ser el soporte de las acciones orientadas al entendimiento y está

\footnotetext{
${ }^{11}$ Vale señalar que en este punto de vincular las prácticas discursivas con el interés por la racionalidad es un ámbito en que Bourdieu debe lidiar con ciertos aspectos concernientes a la relación con lo universal que la teoría habermasiana ya encuentra resueltos de antemano dentro de su matriz teórica. Si la instauración e institucionalización de ámbitos ligados a la racionalidad depende más de la estructuración y distribución de fuerzas presentes en ellos que de la capacidad discursiva, esto deja en entredicho el modo en que el sujeto de la enunciación científica logra obtener su posición y reconocimiento y la defensa que pueda realizar de la vinculación de sus argumentos y valoraciones con algo que vaya más allá de su propia ubicación en el campo. El privilegio argumentativo reclamado para el propio discurso -o para las leyes del propio campoencierra en sí una concesión epistemocéntrica en el sentido que confía en la fuerza argumentativa del discurso científico y en la legitimidad de su conexión con la totalidad del espacio social y las prácticas simbólicas, concesión en que se basa en parte la viabilidad de su propuesta de una pragmática sociológica.
} 
vinculado en forma excluyente con las instituciones estructuradas comunicativamente que tienen a la sociedad civil y la opinión pública como ámbitos propios de realización (cfr: Habermas 1994b). Mientras que para Bourdieu el lenguaje es una institución atravesada por relaciones tanto de comunicación y conocimiento como de poder y dominación, que obtiene gran parte de su rendimiento simbólico debido a la posesión de capitales y la posición institucionalizadas de los hablantes, a la vez que tiene la capacidad de contribuir en forma eficaz a la institucionalización de las acciones y distribuciones que estructuran los diferentes campos.

Respecto de sus respectivas reapropiaciones del pragmatismo lingüístico, a través de ella Habermas busca enmendar los excesos que encuentra en la crítica hiperbólica de la teoría frankfurteana y superar las consecuencias negativas atribuidas a la modernización por Weber, para lo cual recurre a una lectura que hace retroceder las múltiples capacidades de las fuerzas ilocucionarias del lenguaje, señaladas por Austin, en favor del modelo paradigmático de entendimiento y somete la interpretación de Wittgenstein a un patrón consensualista. En tanto que Bourdieu, con el objetivo de resaltar la interdependencia de las funciones simbólicas del lenguaje con las estructuras y dinámicas sociales, por un lado, retoma la gran capacidad realizativa del lenguaje y sus variados modos de empleo presentes en la teoría de los actos de habla -aunque, como hemos visto, incurre en cierta interpretación parcializada de la dimensión ilocucionaria en ella- y, por el otro, se vale del modelo anti-representacionista de cuño wittgensteiniano que conjuga las acciones lingüísticas con las prácticas institucionales y el seguimiento colectivo de reglas. Contra los enfoques comunicacionales y formalistas al estilo habermasiano, Bourdieu otorga prioridad al tratamiento de los mecanismos sociales actuantes en el rendimiento simbólico del lenguaje; es infructuoso aquí tratar de comprender las prácticas lingüísticas sin abarcar sus conexiones y mutuos condicionamientos con el conjunto de las prácticas sociales, las estructuras objetivas y las disposiciones socialmente incorporadas. Bourdieu se reapropia de los núcleos conceptuales de Wittgenstein y Austin de un modo radicalmente distinto al que lo hace Habermas ya que vincula la potencia de la perspectiva pragmática con los condicionamientos y fuerzas sociales que atraviesan las prácticas simbólicas.

La reapropiación pragmática de cada uno pone el acento en esferas muy diversas y da como resultado visiones contrapuestas del lenguaje: o bien como medio por excelencia para la comunicación y el entendimiento o bien como caja de resonancia de las posiciones 
y distribuciones dentro del espacio social y componente fundamental de los procesos de violencia y dominación simbólicas. Por ello, la elección de un basamento teórico u otro implica redimensionar la fuerza pragmática del lenguaje a partir de diferentes condiciones y funciones y otorgarle distintas posibilidades y obstáculos en cada caso. El pensamiento bourdieusiano está en tensión permanente hacia las dinámicas que atraviesan los modos efectivos de producción simbólica, además de que incorpora en forma no secundaria las desigualdades y jerarquías que actúan como vectores y configuran toda práctica lingüística concreta y no toma como paradigma una función del lenguaje, ni una forma de institucionalización, en detrimento de las demás. En este sentido, la potencia heurística propia de los inicios del enfoque pragmático-institucional encuentra en su teoría una continuidad más apropiada que las sucesivas abstracciones a que es sometida en las reflexiones habermasianas tras la separación irreconciliable que éste establece entre los distintos tipos de acción, que hace que los demás usos del lenguaje sólo aparezcan como defectivos a partir del modelo de la acción comunicativa y la función comunicativa del lenguaje quede planteada como modelo y única vía para interacciones humanas válidas. La propuesta de Habermas -si bien presenta ciertos desafíos y diagnósticos que no carecen de interés - toma los rasgos de las distintas esferas de acción exclusivamente desde el criterio de la acción comunicativa y de las pretensiones normativas-reconstructivas que guían su teoría, por lo que los elementos, intereses y funciones de aquellas sólo se presentan como factores obstaculizantes de toda interacción válida antes que como componentes inevitablemente presentes en la producción de variados actos de habla. Desde un enfoque pragmático sería más importante precisar el modo en que las acciones comunicativas están entrelazadas y se relacionan con los factores del contexto -que se constituyen sólo en parte en forma comunicativa-, pero la TAC tiende a obliterar este camino y a considerar los demás factores sólo como entorpecedores de las acciones comunicativas y surgidos de usos desviados o parasitarios; de aquí que termine alejándose del espíritu pragmático, caracterizado por vincular el lenguaje y la racionalidad en forma directa a los múltiples usos y prácticas dados efectivamente en los diferentes ámbitos. ${ }^{12}$

\footnotetext{
${ }^{12}$ La crítica no apunta hacia que Habermas no incorpore esos factores, pues sí los incluye en diversas partes de sus análisis, sino a la explicación que da de ellos, debido a que los ubica en un plano secundario y les otorga sólo funciones distorsionantes; en su pretensión de rehabilitar de modo excluyente la socialización comunicativa anclada en el mundo de vida, no tiene reparos en arrancar sus componentes de todas las demás esferas en que se desenvuelve la vida humana y con ello pierde la posibilidad de ahondar en las interconexiones y procesos similares que se dan entre ambos planos -no sólo negativos o de colonización-.
} 
Si consideramos, siguiendo los presupuestos del giro pragmático, que la fuerza ilocucionaria no deviene de las propiedades internas del lenguaje, sino que se da por la multiplicidad de usos y realizaciones que permite y por su conjugación con factores extralocucionarios -que pueden actuar en forma directa e incardinar expresamente en la intersubjetividad reducida o bien actuar en forma general y funcionar de modo estructural y colectivo en sentido extendido-, entonces todo análisis pragmático-institucionalista debe contemplar las distintas condiciones que hacen posible que el lenguaje posea la eficacia realizativa que le es distintiva. Esta articulación entre diferentes dimensiones es la que da por resultado el establecimiento normativo e institucionalizado de los usos del lenguaje, por ello aquellos abordajes teóricos que prestan especial atención a esta interrelación brindan principios explicativos más enriquecedores y congruentes con la perspectiva pragmática inicial. En definitiva, para lograr un mayor calado en el funcionamiento efectivo del lenguaje es necesario dar cuenta de los variados modos en que el lenguaje es empleado y de la interacción entre los factores locucionarios y extra-locucionarios micro y macro-sociales intervinientes, en esto reside una parte ineludible del legado pragmático y la posibilidad de que un análisis institucionalista pueda retomar fecundamente el potente impulso que introdujo la perspectiva pragmática en los estudios sobre el lenguaje. ${ }^{13}$

\footnotetext{
${ }^{13}$ En este sentido puede entenderse el célebre dictum austiniano acerca de que "ciertamente, pues, el lenguaje ordinario no es la última palabra (...). Pero recordemos, es la primera palabra" (Austin 1962, p. 133): nunca debe dejar de tenerse a las condiciones y usos efectivos del lenguaje como eje de las reflexiones pues no sólo estaremos desaprovechando lo que ellos tienen para ofrecernos, sino que además estaremos tendiendo hacia análisis sesgados desde el punto de vista pragmático.
} 


\section{BIBIBLIOGRAFÍA CITADA}

- $\quad$ Austin, John, 1962, Philosophical Papers, Oxford University Press, Londres.

- $\quad$ Austin, John, 1970, How to do Things with Words, Oxford University Press, Londres.

- Bourdieu, Pierre, 1979, La distinction. Critique sociale du jugement, Minuit, París.

- $\quad$ Bourdieu, Pierre, 1980, Le sens pratique, Minuit, París.

- Bourdieu, Pierre, 1982, Ce que parler veut dire. L'économie des échanges linguistiques, Fayard, París.

- Bourdieu, Pierre, 1987, Choses dites, Minuit, París.

- Bourdieu, Pierre, 1994, Raisons pratiques. Sur la théorie de l'action, Seuil, París.

- Bourdieu, Pierre, 1997, Méditations pascaliennes. Éléments pour une philosophie négative, Seuil, París.

- Bourdieu, Pierre, 2000, Esquisse d'une théorie de la pratique (précédé de Trois études d'ethnologie kabyle), Seuil, París.

- Bourdieu, Pierre, 2001, Langage et pouvoir symbolique, Seuil-Fayard, París.

- Bourdieu, Pierre, 2005, Intelectuales, política y poder, trad. Alicia Gutiérrez, Eudeba, Buenos Aires.

- Calhoun, C., 1993, "Habitus, Field and Capital: The Question of Historical Specificity", en C. Calhoun, E. LiPuma y M. Postone (eds.), Bourdieu: Critical Perspectives, University of Chicago Press, Chicago, pp. 61-88.

- Habermas, Jürgen, 1981, La reconstrucción del materialismo histórico, trad. Jaime Muñiz y Ramón García Cotarelo, Taurus, Madrid.

- Habermas, Jürgen, 1987, Teoría y praxis. Estudios de filosofía social, trad. Salvador Más Torres y Carlos Moya Espí, Tecnos, Madrid.

- Habermas, Jürgen, 1988, La lógica de las ciencias sociales, trad. Manuel Jiménez Redondo, Tecnos, Madrid.

- Habermas, Jürgen, 1990a, Pensamiento postmetafísico, trad. Manuel Jiménez Redondo, Taurus, Madrid.

- Habermas, Jürgen, 1990b, Conocimiento e interés, trad. Manuel Jiménez, José Ivars y Luis Santos, Taurus, Madrid. 
- Habermas, Jürgen, 1994a, Teoría de la acción comunicativa: complemento y estudios previos, $2^{\mathrm{a}}$ ed., trad. Manuel Jiménez Redondo, Cátedra, Madrid.

- Habermas, Jürgen, 1994b, Historia y crítica de la opinión pública. La transformación estructural de la vida pública, $4^{\mathrm{a}}$ ed., trad. Antoni Doménech, Gustavo Gili, Barcelona.

- Habermas, Jürgen, 1998, Facticidad y validez. Sobre el derecho y el Estado democrático del derecho en términos de teoría del discurso, trad. Manuel Jiménez Redondo, Trotta, Madrid.

- Habermas, Jürgen, 2000, Perfiles filosófico-políticos, trad. Manuel Jiménez Redondo, Taurus, Madrid.

- Habermas, Jürgen, 2003a, Teoría de la acción comunicativa I. Racionalidad de la acción y racionalización social, $4^{\mathrm{a}}$ ed., trad. Manuel Jiménez Redondo, Taurus, Madrid.

- Habermas, Jürgen, 2003b, Teoría de la acción comunicativa II. Crítica de la razón funcionalista, $4^{\mathrm{a}}$ ed., trad. Manuel Jiménez Redondo, Taurus, Madrid.

- Habermas, Jürgen, 2003c, Acción comunicativa y razón sin trascendencia, trad. Pere Fabra Abat, Paidós, Buenos Aires.

- Habermas, Jürgen, 2008, El discurso filosófico de la modernidad, trad. Manuel Jiménez Redondo, Katz, Buenos Aires.

- Honneth, Axel, McCarthy, Thomas, Offe, Claus y Wellmer, Albercht (ed.), 1992a, Philosophical Interventions in the Unfinished Project of Enlightenment, MIT Press, Cambridge (Massachusetts) and London.

- Honneth, Axel, McCarthy, Thomas, Offe, Claus y Wellmer, Albercht (ed.), 1992b, CulturalPolitical Interventions in the Unfinished Project of Enlightenment, MIT Press, Cambridge (Massachusetts) and London.

- Passerin, Maurizio y Benhabib, Seyla (ed.), 1997, Habermas and Unfinished Project of Modernity. Critical Essays on The Philosophical Discourse of Modernity, MIT Press, Cambridge (Massachusetts).

- Taylor, Charles, 1995, "To follow a rule", en Philosophical Arguments, Harvard University Press, Cambridge (Massachusetts), pp. 165-180.

- Thompson, John y Held, David (ed.), 1982, Habermas - Critical Debates, Macmillan, London.

- Wittgenstein, Ludwig, 1991, Sobre la certeza, $2^{\mathrm{a}}$ ed., trad. Josep Prades y Vincent Raga, Gedisa, Barcelona.

- Wittgenstein, Ludwig, 2004, Investigaciones filosóficas, $3^{\mathrm{a}}$ ed., trad. Alfonso García Suárez y Ulises Moulines, UNAM/Crítica, Barcelona. 